\title{
Intrahepatic portosystemic shunt in a young female: Views from a developing country
}

\author{
Zain Majid ${ }^{1}$, Muhammad Manzoor ul Haque ${ }^{1}$, Muhammad Danish Ashraf', \\ Nasir Hassan Luck ${ }^{1}$, Ameet Kumar Lalwani ${ }^{2}$ \\ 'Department of Hepatogastroenterology, Sindh Institute of Urology and Transplantation (SIUT), \\ Karachi, Pakistan; \\ ${ }^{2}$ Department of Radiology, Sindh Institute of Urology and Transplantation (SIUT), Karachi, Pakistan
}

\section{ABSTRACT}

A young female presented to us with abdominal distension along with pedal edema. She had no prior medical or surgical history apart from a caesarean section done a few years prior. Initial workup showed low hemoglobin, low serum albumin and slight raised ESR. Her LFTs were slightly deranged. Ultrasound abdomen had evidence of portal hypertension along with splenomegaly. While ultrasound hepatic Doppler revealed a portosystemic shunt between the portal vein and the left hepatic vein, with a shunt ratio of $7.1 \%$. CT scan abdomen confirmed these findings and a diagnosis of Type III intrahepatic portosystemic shunt and spleno-renal shunt was made. Since the patient was currently asymptomatic, she was advised regular follow-ups and was managed conservatively.

Key words: Portal hypertension, intrahepatic portosystemic shunt, congenital portosystemic shunt

\section{INTRODUCTION}

Congential portosystemic shunts occur in 1 in 30,000 children ${ }^{[1]}$ and are due to the persistence of the fetal circulation or due to the embryonic changes. These are rare vascular anomalies, which are also associated with Down's Syndrome.

\section{CASE HISTORY}

Address for Correspondence:

Hepatogastroenterology, Sindh Institute of Urology and Transplantation (SIUT), Karachi, Pakistan

E-mail: Zain88@hotmail.com

\begin{tabular}{|l|}
\hline Access this article online \\
\hline Website: \\
www.intern-med.com \\
\hline DOI: \\
10.2478/jtim-2019-0017 \\
\hline Quick Response Code: \\
\hline \\
\hline \\
\hline
\end{tabular}

A 20-year-old female, having no prior co-morbids, presented to our outpatients' department with the complains of abdominal distension and swelling of her feet since the past 3 months. She had a history of caesarean section, which was done during her last child birth, 3 years ago. There was no history of liver disease, diabetes mellitus, abdominal trauma, use of oral contraceptive pills, alcohol intake, changes in her mental status or the use of any over the counter medications. Her general physical examination was unremarkable.
Her initial workup showed her viral markers to be negative (HbsAg, anti HCV). Laboratory investigations were all normal apart from a low serum hemoglobin $(9.1 \mathrm{~g} / \mathrm{dL})$, low serum albumin $(2.8 \mathrm{~g} / \mathrm{dL})$ and a slightly raised ESR $(25 \mathrm{~mm} / \mathrm{h})$. Her liver function tests were slightly deranged with a total bilirubin $0.93 \mathrm{IU} / \mathrm{L}$, direct bilirubin $0.29 \mathrm{IU} / \mathrm{L}$, alkaline phosphatase (ALP) $144 \mathrm{IU} / \mathrm{L}$, alanine aminotransferase (SGPT) $77 \mathrm{IU} / \mathrm{L}$, aspartate aminotransferase (SGOT) $55 \mathrm{IU} / \mathrm{L}$ and gamma-glutamyl transferase (GGT) 18 IU/L.

Ultrasound abdomen had shown evidence of portal hypertension with splenomegaly.

We proceeded with an ultrasound hepatic doppler, in which the liver was found to have a heterogenous texture with multiple hypoechoic areas scattered in both lobes. Hepatic vein, portal vein and hepatic arteries were all normal and patent. A portosystemic shunt was noted between the portal vein and the left hepatic vein, with a shunt ration being $7.1 \%$. No signs of free fluid was evident. 
In order to confirm her diagnosis, we proceeded with a computed tomography (CT Scan) chest abdomen and pelvis, which showed a liver of $13 \mathrm{~cm}$ having regular margins with no space occupying lesions noted. However, the portal vein was dilated though patent and had a tortuous intrahepatic part of its left branch, having an anomalous communication between the left branch of hepatic vein (Figure 1), representative of a type III intrahepatic portosystemic shunt. A splenorenal shunt was also noted between the splenic vein and left renal vein (Figures 2 and 3). And multiple collaterals at the splenic hilum were seen (Figure 2). A few sub centimeter paraaortic lymph nodes were also observed. No Ascites was noted.

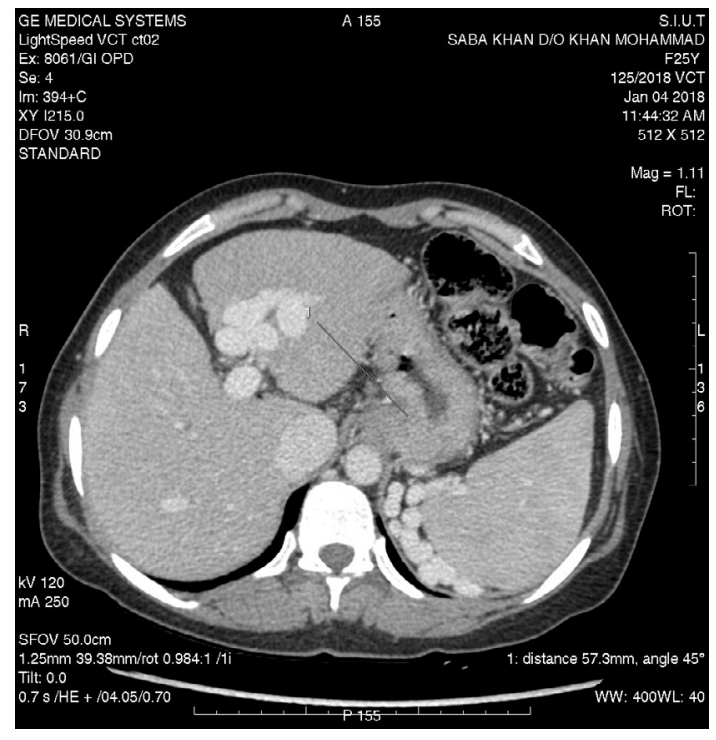

Since the patient was asymptomatic, having no current or prior history of any ailments, we decided to manage her conservatively and she was kept on low dose lactulose along with iron supplements and advised to follow-up regularly.

\section{DISCUSSION}

Congenital portosystemic shunts are commonly divided as either intrahepatic or extrahepatic. ${ }^{[2]}$ Their clinical presentation is variable, ranging from being either asymptomatic to having severe organ damage. ${ }^{[2]}$ The extrahepatic shunts unlike their intrahepatic counterparts are associated with focal nodular hyperplasia, encephalopathy

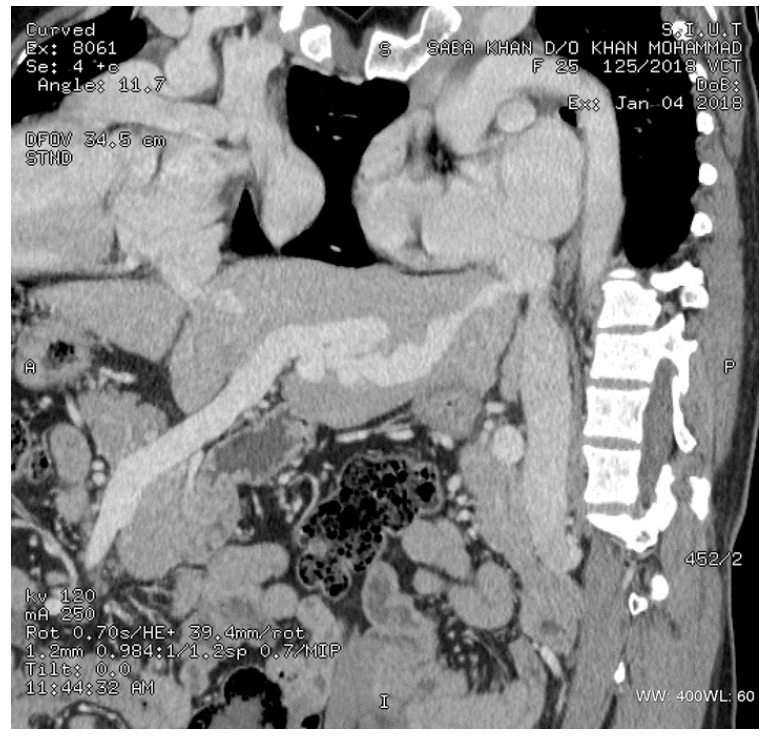

Figure 1: MDCT axial and coronal reconstructed images in portovenous phase showing abnormal anomalous communication between left branch of portal vein and left hepatic vein. MDCT: multi-detector computed tomography.

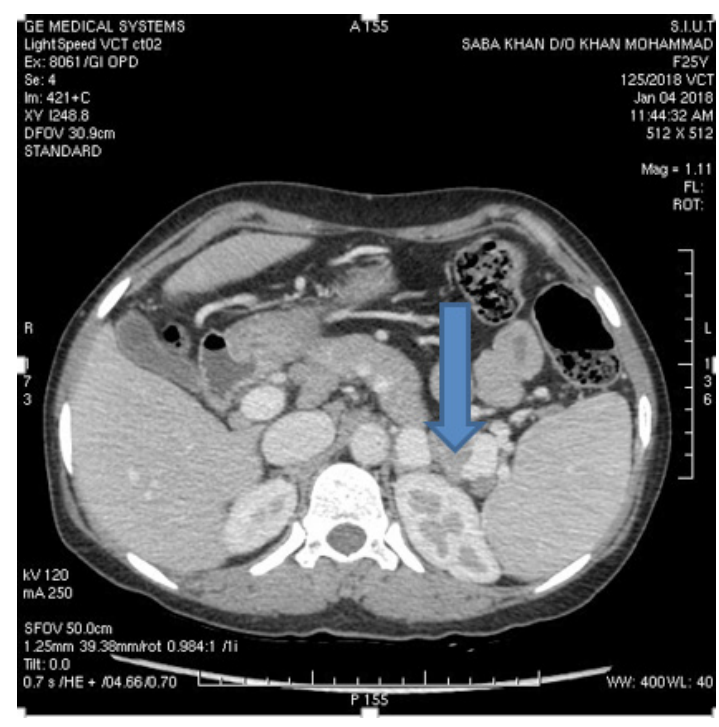

Figure 2: MDCT axial image in portovenous phase showing collaterals at splenic hilum with the formation of splenorenal shunt (arrow pointed). MDCT: multi-detector computed tomography.

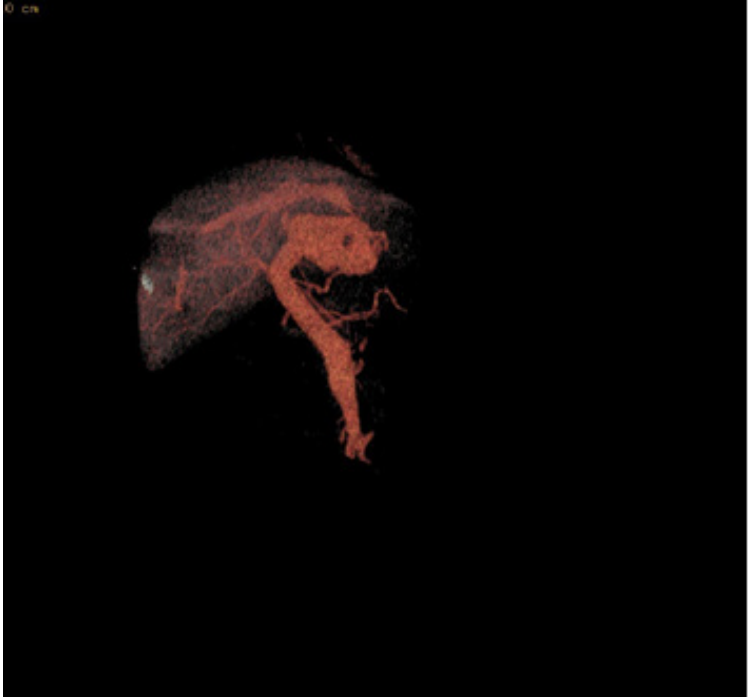

Figure 3: Selected virtual reality images showing anomalous communication between left hepatic vein and left portal vein 
and regenerative nodule. ${ }^{[3]}$ While the intrahepatic shunts are mostly due to blunt trauma or occur in Budd Chiari Syndrome. ${ }^{[3]}$ No such cause could be identified in our patient. These shunts are divided into three types depending upon its course; the paraumbilical, the inferior vena cava type and a miscellaneous one. ${ }^{[4]}$

Congenital portosystemic shunts are usually diagnosed on imaging, with a doppler ultrasound characterizing their nature and are used to calculate the shunt ratio. A shunt ratio which is of more than $60 \%$ needs to be treated in order to prevent further complications. ${ }^{[5]}$ In our case, the shunt ratio was very low (7.1\%). Magnetic resonant imaging (MRI) shows flow voids in the shunt region, while a transhepatic portography is the diagnostic test of choice to demonstrate the shunt. ${ }^{[6,7]}$

The treatment of choice for these shunts include transcatheter embolization and the use of surgical procedure like hepatic resection, shunt ligation and creating an alternative portosystemic shunt. ${ }^{[8]}$

\section{CONCLUSION}

Portosystemic shunts, even though are of rare occurrence, need to be identified and managed accordingly. Our case highlights the importance of imaging in such patients and the need of conservative management with a regular follow-up when these patients present without any symptoms.

\section{Conflict of Interest}

None declared.

\section{REFERENCES}

1. Bernard O, Franchi-Abella S, Branchereau S, Pariente D, Gauthier F, Jacquemin E. Congenital portosystemic shunts in children: recognition, evaluation, and management. Semin Liver Dis 2012; 32:273-87.

2. Timpanaro T, Passanisi S, Sauna A, Trombatore C, Pennisi M, Petrillo G, et al.Congenital portosystemic shunt: our experience. Case Rep Pediatr 2015; 2015: 691618.

3. Chandrasekharan R, Pullara SK, Thomas T, Kader NP, Moorthy S. Congenital intrahepatic portosystemic shunts: Imaging findings and endovascular management. Indian J Radiol Imaging 2016; 26: 92-4.

4. Itai Y, Saida Y, Irie T, Kajitani M, Tanaka YO, Tohno E. Intrahepatic portosystemic venous shunts: spectrum of CT findings in external and internal subtypes. J Comput Assist Tomogr 2001; 25: 348-54.

5. Tsitouridis I, Sotiriadis C, Michaelides M, Dimarelos V, Tsitouridis K, Stratilati S. Intrahepatic portosystemic venous shunts: Radiological evaluation. Diagn Interv Radiol 2009; 15: 182-7.

6. Araki T, Ohtomo K, Kachi K, Monzawa S, Hihara T, Ohba H, et al. Magnetic resonance imaging of macroscopic intrahepatic portosystemic venous shunts. Gastrointest Radiol 1991; 16: 221-4.

7. Pocha C, Maliakkal B. Spontaneous intrahepatic portal-systemic venous shunt in the adult: case report and review of the literature. Dig Dis Sci 2004; 49: 1201-6.

8. Araki T, Konishi T, Yasuda S, Osada T, Araki T. Embolization of the patent ductus venosus in an adult patient. AJR Am J Roentgenol 2003; 180:716-8.

How to cite this article: Majid Z, Manzoor ul Haque M, Danish Ashraf M, Luck NH, Lalwani AK. Intrahepatic portosystemic shunt in a young female: Views from a developing country. J Transl Int Med 2019; 7: 118-20. 